\title{
Development and Evaluation of Chitosan Based Polymeric Nanoparticles of an Antiulcer Drug Lansoprazole
}

\author{
E. Nagarajan ${ }^{\mathrm{a}}$, P. Shanmugasundaram ${ }^{\mathrm{a}}$, V. Ravichandiran ${ }^{\mathrm{a}}$, A. Vijayalakshmi ${ }^{\mathrm{a}}$, B. Senthilnathan ${ }^{\mathrm{b}}$, K. Masilamani ${ }^{\mathrm{b} *}$ \\ ${ }^{a}$ School of Pharmaceutical Sciences, VISTAS, Vels University, Pallavaram, Chennai-117, Tamilnadu, India. ${ }^{b}$ Department of Pharmaceutics, College of \\ Pharmacy, Jaya College of Paramedical Sciences, Thiruninravur, Tiruvallur. 'Department of Pharmaceutical Engineering, University Malaysia Pahang, \\ Lebuhraya Tun Razak 26300 Gambang, Kuantan Pahang Darul Makmur.
}

\begin{tabular}{l} 
ARTICLE INFO \\
\hline Article history: \\
Received on: 19/01/2015 \\
Revised on: 18/02/2015 \\
Accepted on: 03/03/2015 \\
Available online: $27 / 04 / 2015$ \\
\hline Key words: \\
Lansoprazole, polymeric \\
nanoparticles, chitosan, \\
ionotropic gelation
\end{tabular}

\begin{abstract}
Objective: The development of new delivery systems for the controlled release of drugs is one of the most innovative fields of research in pharmaceutical sciences. Nanoparticles specially designed to release the drug in the vicinity of target sites. The aim of this study was to formulate and evaluate the Lansoprazole nanoparticles to improve the therapeutic efficacy of Lansoprazole by loading in nanoparticle drug delivery system.

Materials and Methods: Lansoprazole loaded chitosan nanoparticles (CNP1 to CNP10) were prepared by ionotropic gelation method. The formulated nanoparticles were evaluated for external morphological characters, determination of particle size analysis, zeta potential, drug content, entrapment efficiency and in-vitro release studies.

Results: The drug content for the Lansoprazole loaded chitosan nanoparticles varied from $69.5 \pm 7.2 \%$ to $87.9 \pm 1.2 \%$. The entrapment efficiencies were found to be minimum and maximum of $39.3 \pm 2.6 \%$ and $85.6 \pm 1.2 \%$, the optimum entrapment efficiency was found to be $85.3 \pm 0.8 \%$, particle size varied from $360 \pm 12 \mathrm{~nm}$ to $814 \pm 62 \mathrm{~nm}$, zeta potential values were in positive and increased from $11.2 \pm 1.2 \mathrm{mV}$ to $18.7 \pm 0.4 \mathrm{mV}$. In-vitro release of drug follows zero order and showed sustained release behavior for a period of $24 \mathrm{hr}$.

Conclusion: The study demonstrated the successful preparation of sustained release polymeric nanoparticles of Lansoprazole.
\end{abstract}

\section{INTRODUCTION}

During last two decades, considerable attention has been given to the development of novel drug delivery system (NDDS) (Santhi et al., 2005). The rational for control drug delivery is to alter the pharmacokinetics and pharmacodynamics of drug substance in order to improve the therapeutic efficacy and safety through the use of novel drug delivery system. Besides more traditional matrix or reservoir drug delivery system, colloidal drug delivery system has gained in popularity. The major colloidal drug delivery system includes liposome and polymeric nanoparticles. These systems have been investigated primarily for site specific drug delivery, for controlled drug delivery, and also for the enhancement of dissolution rate/bioavailability of poorly water-soluble drugs. Nanoparticles are defined as particulate dispersions or solid particles with a size

\footnotetext{
* Corresponding Author

E-Mail: masilamani33@gmail.com
}

in the range of 10-1000 $\mathrm{nm}$. Nanoparticles are sub-nano sized colloidal structures composed of synthetic or semi synthetic polymers. They offer advantages like increased bioavailability, site specific drug delivery, sustained release of drug over longer period of time, retention of dosage form in entire length of gastrointestinal tract and convenient to patient due to reduction in frequent dosing (Chen et al., 2001).

Lansoprazole is a drug commonly used in the management of peptic ulcer in various conditions such as acidrelated disorders of the upper GIT, Gastro Oesophageal Reflux Disease, benign gastric ulcer \& duodenal ulcer, treatment of NSAID-associated benign gastric ulcers, duodenal ulcers and long term management Zollinger-Ellison syndrome (Matheson and Jarvis, 2001). This drug is primarily metabolized by liver.

Hence it is a need to reduce the dose to the hepatic and renal failure patients. But reduced dose in conventional systems may not produce required pharmacological effect. Further the gastro-intestinal tract is exposed to different $\mathrm{pH}$ environment, which the Lansoprazole may be wasted. 
This drug, on regular usage, is reported to cause adverse effects including abdominal pain, diarrhoea, dizziness, skin rashes, angioedema, thrombocytopenia, impotence etc. Due to its severe side effects, controlled delivery-nanoparticles of Lansoprazole at optimal concentration may be required (Shimizu et al., 2003). Comparing to other routes, oral route is preferable with respect to safety, comfort and reliability. Hence controlled delivery of Lansoprazole by oral route is ideal. Controlled release of Lansoprazole from nanoparticles will reduce the frequency of dosing and dose size and may increase patient convenience.

Chitosan exhibits many advantages in developing nanoparticles, including biocompatibility, biodegradability, and low-immunogenicity. The high positive charge density also confers its mucoadhesive properties and makes it an ideal candidate for the delivery of drugs to mucosal tissues. Chitosan also has a very low toxicity. Its $\mathrm{LD}_{50}$ in laboratory mice is $16 \mathrm{~g} / \mathrm{kg}$ body weight, which is close to sugar and salt (Yung-Chih Kuo, 2005).

So the aim of the present study was to formulate gastroretentive nanoparticles of Lansoprazole to deliver the drug at controlled rate to its absorptive site so that its oral bioavailability can be enhanced.

\section{MATERIALS AND METHODS}

\section{Materials}

Lansoprazole were obtained from Tablets India Ltd., Chennai. Chitosan and Sodium tripolyphospate were purchased from Chemika-Biochemika-Reagents, Mumbai. All other chemicals and reagents used were of Analytical grade.

\section{Method}

\section{Drug polymer compatibility studies}

Compatibility of drug with excipients was determined by carrying out FTIR studies (Sonavane and Devarajan, 2007). IR spectra of pure Lansoprazole, additives and combination of Lansoprazole with additives were obtained by using Perkin-Elmer Fourier transform infrared spectrophotometer using $\mathrm{KBr}$ pellets. For DSC studies Lansoprazole, additives and combination of Lansoprazole with additives were sealed in aluminum pans and the DSC thermograms were recorded at a heating rate of $10^{\circ} / \mathrm{min}$.

\section{Formulation of Lansoprazole nanoparticles \\ Preparation of capsule of modified solubility to protect the drug from degradation}

To protect the drug from degradation for first $2 \mathrm{hr}$, a novel concept, by making insoluble body of capsule was adopted for filling the Lansoprazole nanoparticles. The solubility of the hard gelatin capsules were modified by exposing the capsules to the formaldehyde vapors produced by the reaction between formaldehyde solution and potassium permanganate. To achieve this, capsules were placed on a wire mesh and kept inside a desiccator containing formaldehyde $15 \%$ and potassium permanganate. The vapors of formaldehyde formed were exposed to capsule until the capsule with sufficient solubility was achieved. Then the capsules were removed and dried at $50{ }^{\circ} \mathrm{C}$ to ensure completion of reaction between formaldehyde and gelatin. Finally the capsules were dried at room temperature to remove the excess of formaldehyde vapor (Sutch et al., 2003 \& McConvillea et al., 2004).

\section{Formulation of Lansoprazole loaded chitosan nanoparticles}

Lansoprazole loaded chitosan nanoparticles were prepared by ionotropic gelation technique (Agnihotri et al., 2004). Chitosan solution was prepared by dissolving various concentrations of chitosan $(0.1$ to $1 \%)$ in $100 \mathrm{ml}$ of $1 \% \mathrm{v} / \mathrm{v}$ acetic acid. $0.1 \%$ TPP solution was prepared by dissolving $100 \mathrm{mg}$ of TPP in $100 \mathrm{ml}$ of deionized water. $100 \mathrm{mg}$ of Lansoprazole was added to TPP solution.

The solution was stirred for $1500 \mathrm{rpm}$ for $30 \mathrm{~min}$ on ultrasonicator (vibronics) and TPP solution was added drop wise and kept stirring for 3 hours on homogenizer. Nanoparticles were obtained upon the addition of a TPP aqueous solution to a chitosan solution. The NP suspension is then centrifuged at 15,000 rpm for $10 \mathrm{~min}$ using high-speed centrifuge (Sigma). The formation of nanoparticles results in interaction between the negative groups of TPP and the positively charged amino groups of chitosan.

\section{Evaluation of Lansoprazole nanoparticles}

The nanoparticles containing Lansoprazole were evaluated by studying various physicochemical properties.

\section{Drug Content}

The drug content in each formulation was determined by weighing nanoparticles equivalent to $30 \mathrm{mg}$ of Lansoprazole and dissolving in $100 \mathrm{ml}$ of $6.8 \mathrm{pH}$ phosphate buffer, followed by stirring. The solution was filtered through a $0.45 \mu$ membrane filter, diluted suitably and the absorbance of resultant solution was measured spectrophotometrically at $285 \mathrm{~nm}$ using $6.8 \mathrm{pH}$ phosphate buffer as blank. The drug content of the prepared nanoparticles was determined by the formula:

Drug content $(\%)=\underline{\text { Weight of drug in nanoparticles }} \times 100$

Weight of nanoparticles

\section{Drug Entrapment Efficiency}

The entrapment efficiency is also known as Association Efficiency. The drug loaded nanoparticles were centrifuged at a high speed of 3500-4000 rpm for $30 \mathrm{~min}$ and the supernatant is assayed for non-bound drug concentration by UV spectrophotometer (Das et al., 2005). The percentage Drug Entrapment Efficiency (DEE) was calculated as follows:

DEE $\%=\underline{\text { Amount of drug actually present }} \times 100$

Theoretical drug load expected 


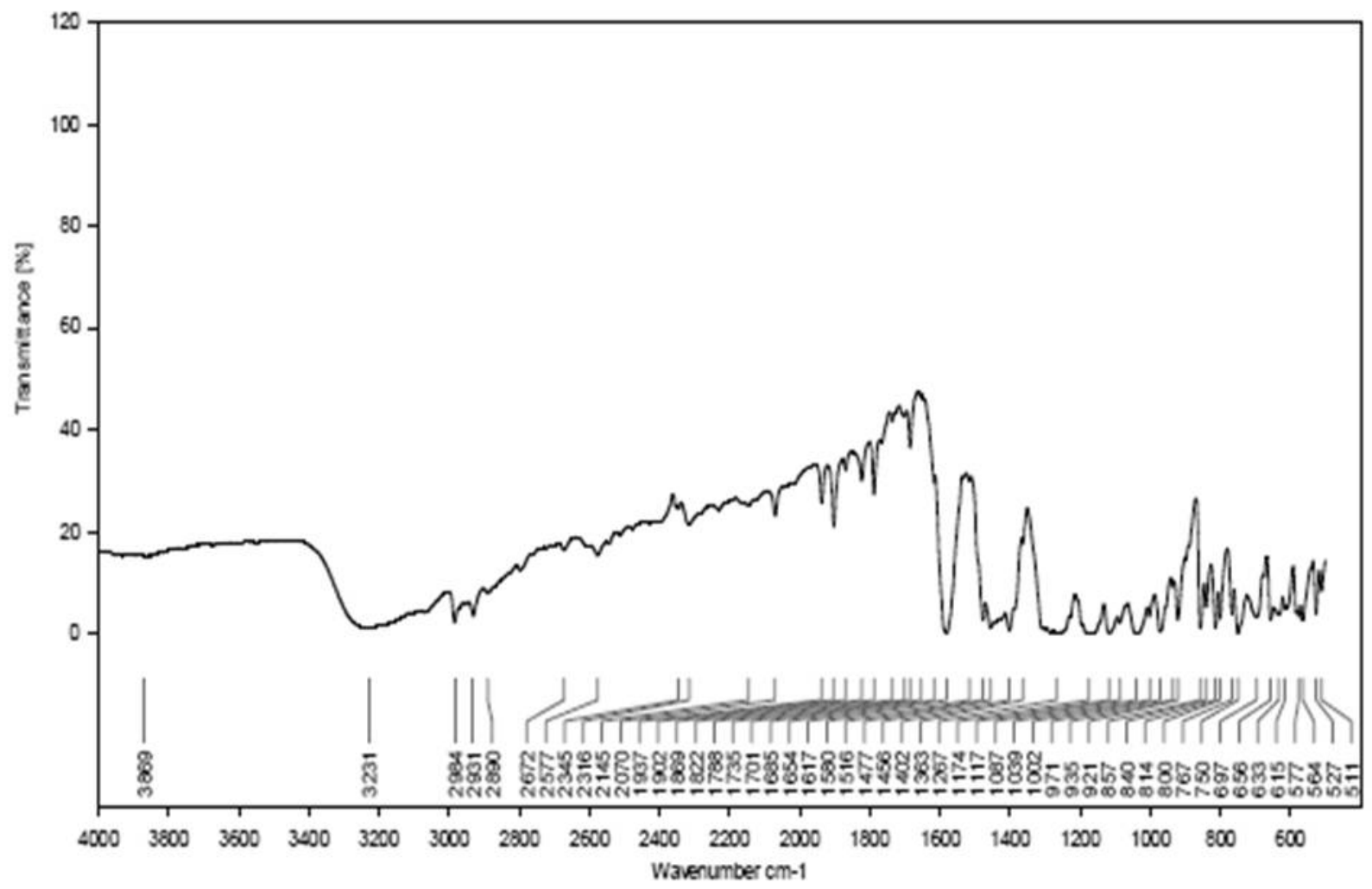

Fig. 1: FT-IR spectra of pure Lansoprazole.

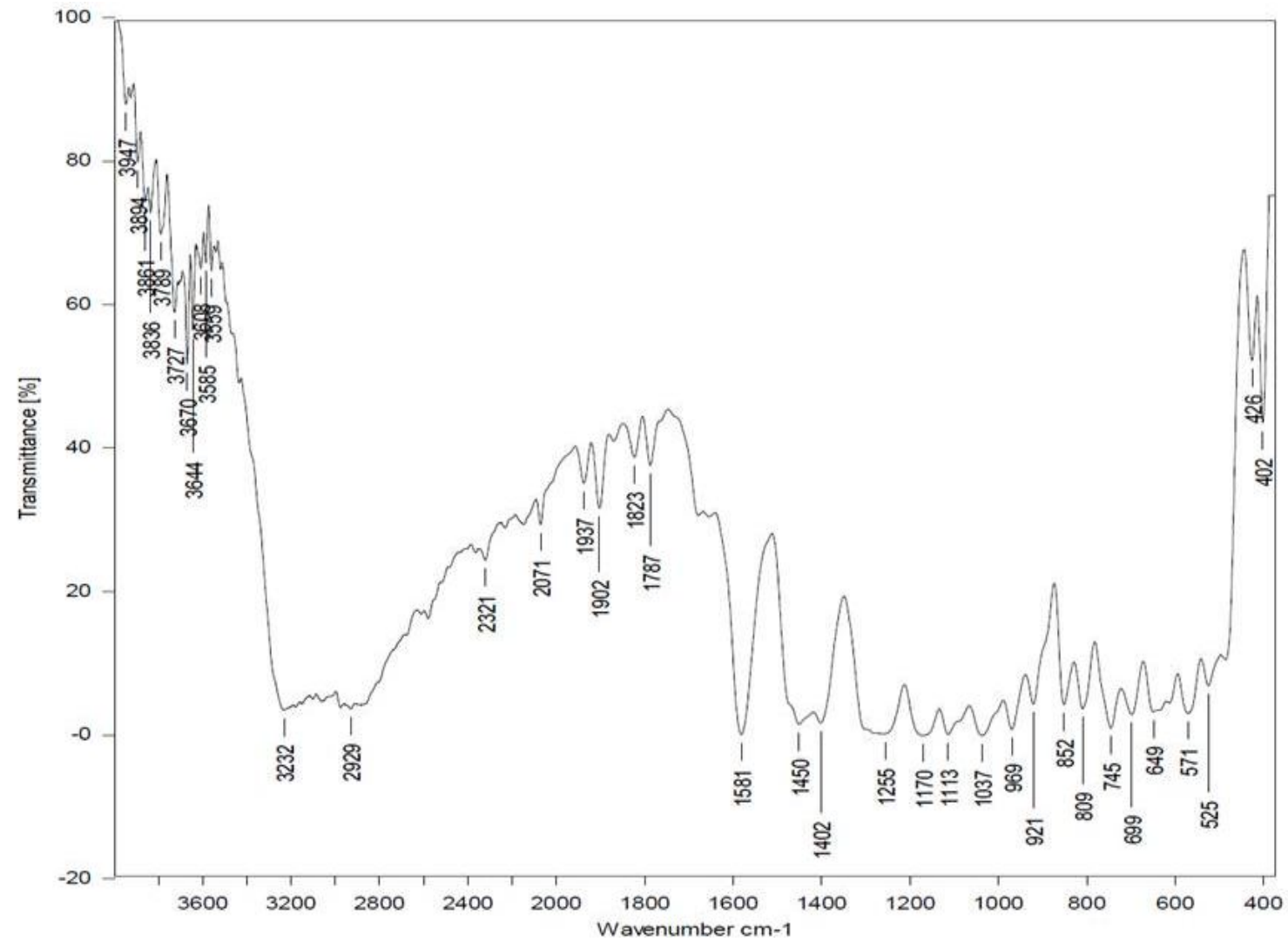

Fig. 2: FT-IR spectra of Lansoprazole loaded nanoparticles. 


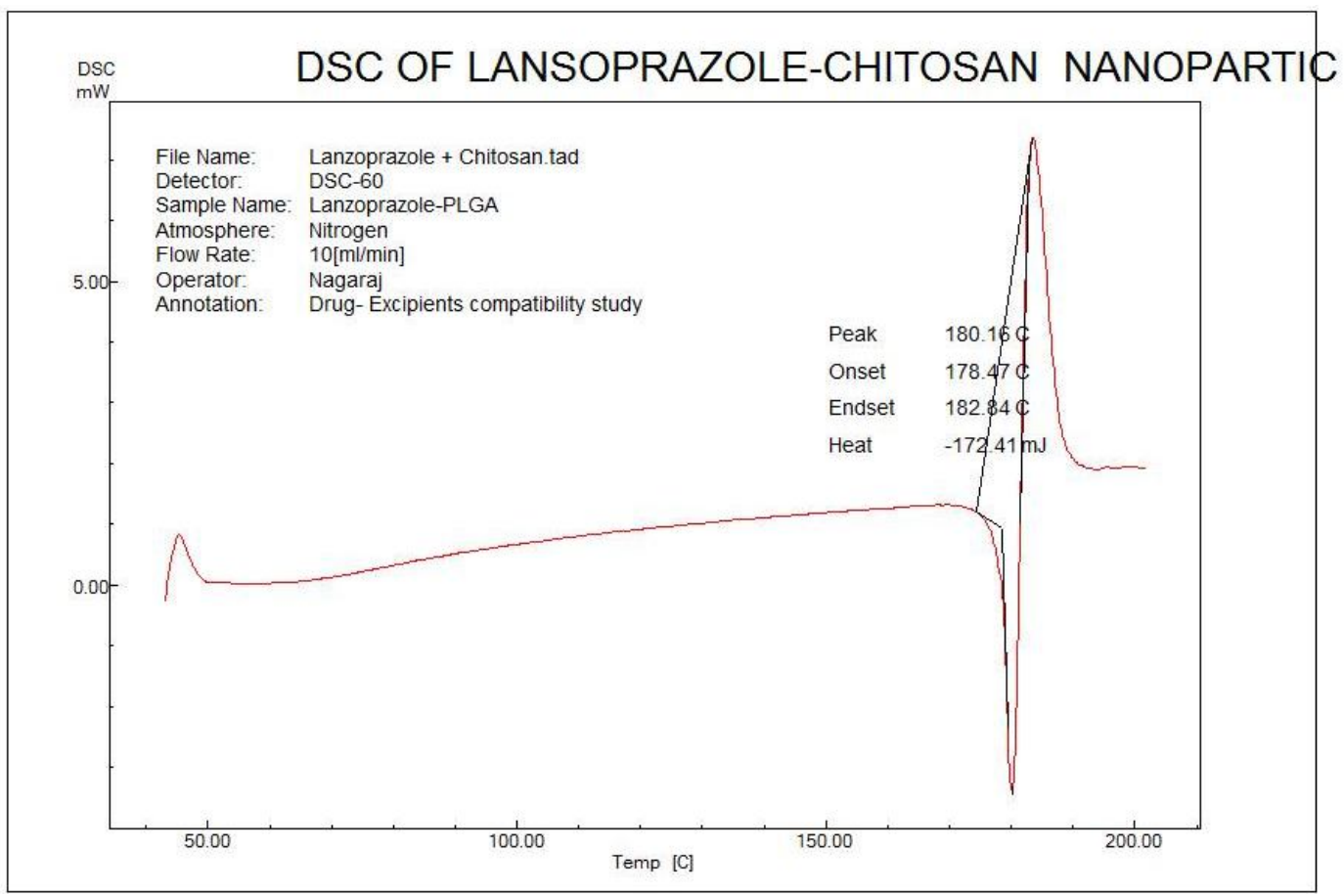

Fig. 3: DSC of Lansoprazole-chitosan nanoparticles.

\section{Particle Size and Surface Charge}

The particle size analysis and zeta-potential measurement were analysed by Zetasizer Nano ZS (Malvern Instruments, UK). For the analysis, the nanoparticle sample of the desired concentration was flushed through a folded capillary cell (DTS1060) and the measurement was carried out on the second filling; a sufficient sample volume was used to completely cover the electrodes of the cell. The sample was injected slowly and analysis was carried out if there were no visible air bubble inclusions present. After inspection, the cell was placed into the Zetasizer and equilibrated at for $2 \mathrm{~min}$ prior to the particle size measurements, of which there were six replicates (Elshafey et al., 2010).

\section{Transmission Electron Microscopy (TEM)}

The morphology studies of the Lansoprazole nanoparticles were studied using transmission electron spectroscopy set at $200 \mathrm{kV}$ by placing an air dried nanoparticle suspension on copper electron microscopy grids (Arias et al., 2010).

\section{In-vitro Release Studies}

Dissolution studies were carried out by using USP dissolution test apparatus (Kalantzi et al., 2009). Capsule filled with nanoparticles equivalent to $30 \mathrm{mg}$ of Lansoprazole was placed in dissolution media in dissolution apparatus. In order to simulate the $\mathrm{pH}$ changes along the GI tract, three dissolution media with $\mathrm{pH} 1.2,7.4$ and 6.8 were sequentially used referred to as sequential $\mathrm{pH}$ change method. When performing experiments, the $\mathrm{pH} 1.2$ medium was first used for 2 hours (since the average gastric emptying time is $2 \mathrm{hrs}$.), then removed and the fresh $\mathrm{pH} 7.4$ phosphate buffer saline was added. After 3 hours (average small intestinal transit time is 3 hrs.), then the medium was removed and colonic fluid $\mathrm{pH} 6.8$ buffer was added for subsequent hours. $900 \mathrm{ml}$ of the dissolution medium was used at each time. Rotation speed was $100 \mathrm{rpm}$ and temperature was maintained at $37 \pm 0.5{ }^{\circ} \mathrm{C} .5 \mathrm{ml}$ of dissolution media was withdrawn at predetermined time intervals and fresh dissolution media was replaced. The samples were withdrawn at specified intervals and analyzed at $285 \mathrm{~nm}$ by UV absorption spectroscopy and the cumulative percentage release was calculated over the sampling times (Paulo and Jose, 2001).

\section{Statistical analysis}

The results were expressed as mean \pm standard deviation $(\mathrm{SD}, \mathrm{n}=3)$. The data were statistically analyzed by one-way analysis of variance using Graph Pad Instat ${ }^{\circledR}$, version 3.05 (USA), and significant difference was set at $\mathrm{p}<0.05$.

\section{RESULTS AND DISCUSSION}

\section{Drug-Excipient Compatibility Studies IR and DSC analysis}

The characteristic absorption peaks of Lansoprazole appeared at 3231, $2984 \&$ \& 2930, 1580, 1282, 1118 denoting stretching vibration of $-\mathrm{NH}-,-\mathrm{CH}_{2}$, aromatic ring, $\mathrm{C}-\mathrm{O}$ and ether bond, respectively. These characteristic bands were observed in all of the recorded IR spectra. The DSC thermogram revealed that the additives showed superimposition on thermogram, however mild preshift was observed. The FTIR and DSC results revealed that there was no interaction between the drug and additives used in the formulation. The IR and DSC were shown from Fig. 1 to Fig. 3. 


\section{Preparation of Lansoprazole nanoparticles}

Lansoprazole loaded chitosan nanoparticles were prepared as per the formula given in Table 1 and the prepared nanoparticles were visually observed. The particles obtained were of smooth and free flowing. Lansoprazole loaded chitosan nanoparticles were prepared by ionic gelation technique. The chitosan nanoparticles were prepared based on the ionic interaction of a positively charged chitosan solution and negatively charged TPP solution. The charge density of both chitosan and TPP solution has a great effect on the ionic interaction. No visible impurity was seen in both chitosan nanoparticles.

\section{Drug content}

The drug content for the Lansoprazole loaded chitosan nanoparticles varied from $69.5 \pm 7.2 \%$ to $87.9 \pm 1.2 \%$. The drug content was decreased with increase in chitosan concentration. This may be due to loss of drug during manufacturing stage or increase in entrapment efficiency, so that drug is not available for estimation. This result indicated that there was no drug loss by manufacturing process or by excipients used in the formulation.

\section{Entrapment efficiency}

The entrapment efficiency of chitosan nanoparticles increased with increase in polymer concentration upto $0.5 \%$ chitosan (CNP5). After that, there was no significant increase in entrapment efficiency. This may due to unavailability of drug for entrapment. The entrapment efficiencies were found to be minimum and maximum of $39.3 \pm 2.6 \%$ and $85.6 \pm 1.2 \%$, for chitosan. But the maximum entrapment efficiency was not considered as optimum. The optimum percentage efficiency was based on the drug content and polymer usage. The optimum entrapment efficiency was found to be $85.3 \pm 0.8 \%$ and $95.5 \pm 0.6 \%$ for chitosan. From drug content and entrapment efficiency results chitosan nanoparticles CNP5 were considered as optimum trials.

\section{The particle size}

The particle size and size distribution are the most important characteristics of nanoparticles systems. Many studies have demonstrated that nanoparticles of sub-micron size have a number of advantages over microparticles as a drug delivery system (Schubert and Muler-Goyman, 2003). The particle size of chitosan nanoparticles varied from $360 \pm 12 \mathrm{~nm}$ to $814 \pm 62 \mathrm{~nm}$.
The mean particle size of chitosan nanoparticles was reduced from CNP1 (620 $\pm 42 \mathrm{~nm})$ to CNP5 (360 $\pm 12 \mathrm{~nm})$ with increase in polymer concentration. This may be due to avoidance of aggregation of drug particles. But the particle size was increased from CNP5 $(360 \pm 12 \mathrm{~nm})$ to $\mathrm{CNP} 10(814 \pm 62 \mathrm{~nm})$ due to increase in polymer concentration. The zeta potential values of chitosan nanoparticles were in positive and increased from $11.2 \pm 1.2 \mathrm{mV}$ to $18.7 \pm 0.4 \mathrm{mV}$. The positive value was due to the polar nature $\left(\mathrm{NH}_{3}\right.$ group) of chitosan. The CNP5 trial held a value of $18.3 \pm 0.2 \mathrm{mV}$. There was significant increase in zeta potential value from CNP1 to CNP5, but from CNP5 to CNP10 there was no significant increase in zeta potential value. Hence from these studies, formulation CNP5 $(18.3 \pm 0.2 \mathrm{mV})$ was considered as optimum. The results of drug content, entrapment efficiency, particle size and zeta potential for chitosan nanoparticles were tabulated in Table 2 .

\section{In-vitro release studies}

From the in-vitro release studies of Lansoprazole loaded with chitosan nanoparticles (CNP1 - CNP10), it was observed that with all formulation, there was absolutely no drug release in simulated gastric fluid (acidic $\mathrm{pH} 1.2$ ) for initial 2 hours. The drug release was found in simulated intestinal fluid $(\mathrm{pH} 7.4$ phosphate buffer) and in colonic medium (pH 6.8 phosphate buffer). In-vitro release profiles in intestinal/colonic medium were found to have very good controlled efficacy. During dissolution study it was found that, the drug release depends upon the nature of the polymer matrix as well as the $\mathrm{pH}$ of the media. In common, increase in polymer concentration produced much more time for release of drug for all formulations. More concentration of polymer $(\geq 0.6 \%$ chitosan - CNP6 to CNP10) formed slow release of drug for more than $24 \mathrm{hr}$. Less concentration of polymer $(\leq 0.5 \%$ chitosan - CNP1 to CNP4) formed quick release of drug within short period. Hence the formulations (CNP1-CNP4, CNP6CNP10,) were considered to be not satisfactory for controlled delivery of Lansoprazole either by quick release or over retarding. Lansoprazole nanoparticles prepared with $0.5 \%$ chitosan (CNP5) showed controlled and sustained drug release for a period of $24 \mathrm{hr}$. The percentage cumulative drug release of CNP5 at the end of 24 hr was found to be $99.74 \pm 0.26 \%$ (Fig. 5a and 5b). Based on the in-vitro drug release study results of Lansoprazole-Chitosan nanoparticles have shown desirable drug release rates than the formulation prepared with chitosan.

Table 1: Formula for preparation of Lansoprazole loaded Chitosan nanoparticles.

\begin{tabular}{|c|c|c|c|c|c|c|c|c|c|c|c|}
\hline S. No. & Ingredients & CNP1 & CNP2 & CNP3 & CNP4 & CNP5 & CNP6 & CNP7 & CNP8 & CNP9 & CNP10 \\
\hline 1 & Lansoprazole (mg) & 30 & 30 & 30 & 30 & 30 & 30 & 30 & 30 & 30 & 30 \\
\hline 2 & Chitosan (\%) & 0.1 & 0.2 & 0.3 & 0.4 & 0.5 & 0.6 & 0.7 & 0.8 & 0.9 & 1.0 \\
\hline 3 & Tripolyphosphate solution (ml) & 100 & 100 & 100 & 100 & 100 & 100 & 100 & 100 & 100 & 100 \\
\hline 4 & $0.1 \%$ Acetic acid solution $(\mathrm{ml})$ & 100 & 100 & 100 & 100 & 100 & 100 & 100 & 100 & 100 & 100 \\
\hline
\end{tabular}

Table 2: Drug content, entrapment efficiency, particle size and zeta potential for chitosan nanoparticles (CNP1 - CNP10).

\begin{tabular}{lcccccccccc}
\hline Parameters/Trials & CNP1 & CNP2 & CNP3 & CNP4 & CNP5 & CNP6 & CNP7 & CNP8 & CNP 9 & CNP10 \\
\hline Drug content $(\%)$ & $87.9 \pm 1.2$ & $87.7 \pm 2.4$ & $87.6 \pm 2.6$ & $86.7 \pm 3.2$ & $86.3 \pm 0.6$ & $84.3 \pm 2.6$ & $80.4 \pm 3.2$ & $78.3 \pm 6.8$ & $75.8 \pm 6.8$ & $69.5 \pm 7.2$ \\
Entrapment efficiency $(\%)$ & $39.3 \pm 2.6$ & $55.5 \pm 2.4$ & $62.7 \pm 3.7$ & $73.6 \pm 0.8$ & $85.3 \pm 0.8$ & $84.3 \pm 3.6$ & $85.3 \pm 2.9$ & $85.6 \pm 1.2$ & $84.9 \pm 1.4$ & $83.3 \pm 3.6$ \\
Particle Size (nm) & $620 \pm 42$ & $602 \pm 36$ & $580 \pm 42$ & $460 \pm 42$ & $360 \pm 12$ & $480 \pm 78$ & $520 \pm 76$ & $625 \pm 55$ & $710 \pm 82$ & $814 \pm 62$ \\
Zeta potential(mV) & $11.2 \pm 1.2$ & $12.2 \pm 1.3$ & $13.4 \pm 1.4$ & $14.3 \pm 0.6$ & $18.3 \pm 0.2$ & $18.4 \pm 0.4$ & $18.1 \pm 0.5$ & $18.6 \pm 0.2$ & $18.4 \pm 0.2$ & $18.7 \pm 0.4$ \\
\hline
\end{tabular}




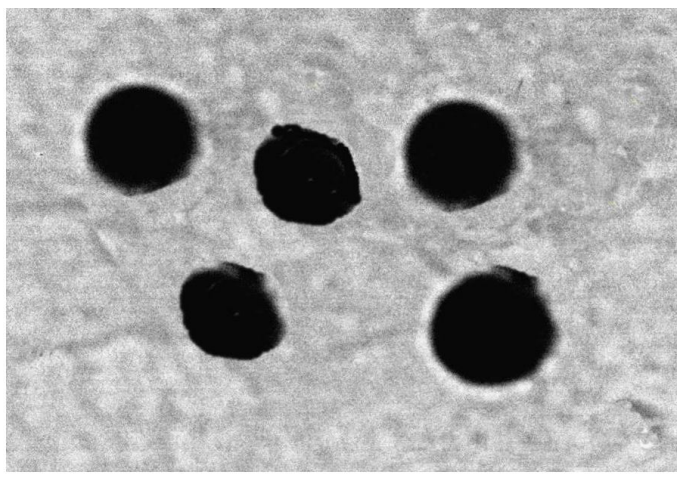

Fig. 4: TEM of Chitosan nanoparticles (CNP5).

\section{\% Cumulative Drug Release (CNP1 -CNP5)}

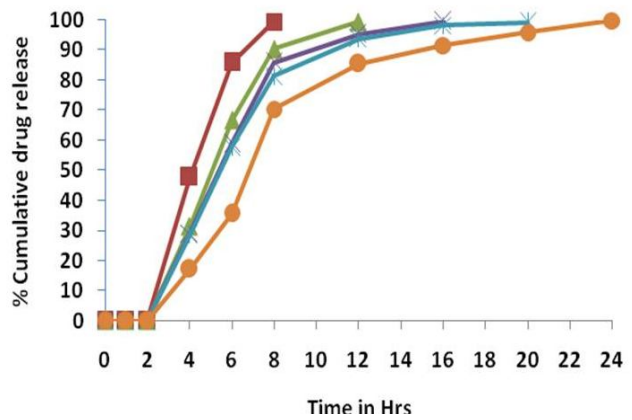

$\rightarrow$ CNP 2

$\leftarrow$ CNP 3

$\because \mathrm{CNP} 4$

- CNP 5

Fig. 5a: In-vitro release of Lansoprazole loaded chitosan nanoparticles (CNP1 to CNP5).

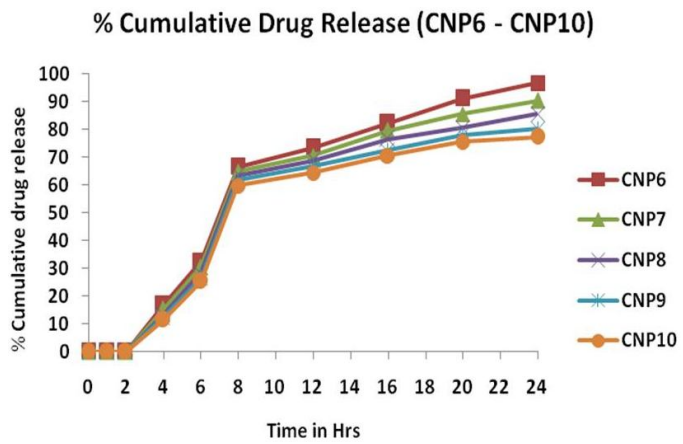

Fig. 5b: In-vitro release of Lansoprazole loaded chitosan nanoparticles (CNP6 to CNP10)

\section{CONCLUSION}

The nanoparticles containing Lansoprazole (CNP5) exhibited most of the ideal characters required for an oral controlled release dosage forms. The nanoparticles (CNP5) of lower particle size $(360 \pm 12 \mathrm{~nm})$ aided with postively charged surface charge $(18.3 \pm 0.2 \mathrm{mV})$ has been achieved. The release profile indicated continuous controlled release up to $24 \mathrm{hr}$. Hence it can be concluded that the newly developed oral controlled drug delivery system - nanoparticles of Lansoprazole is considered to be ideal and effective in the management of ulcer and related conditions.

\section{ACKNOWLEDGEMENT}

Authors acknowledge sincere thanks to the management for the facilities granted for the research work.

\section{REFERENCE}

Agnihotri SA, Mallikarjuna NN, Aminabhavi TM. Recent advances on chitosan-based micro-and nanoparticles in drug delivery. $\mathrm{J}$ Control Release, 2004;100:5-28.

Arias JL, Lopez-viota M, Saez-Fernandez E, Ruiz MA. Formulation and physicochemical characterization of poly (ecaprolactone) nanoparticles loaded with ftorafur and diclofenac sodium. Colloids and Surfaces B: Biointerfaces. 2010; 75(1):204-208.

Chen DB, Yang T Z, Lu W L, Zhang Q. Invitro and in vivo study of two types of long circulating solid lipid nanoparticles containing pacitaxel, Chem. Pharm. Bull., 2001; 49: 1444-1447.

Das S, Banerje R, Belare J. Aspirin loaded albumin nanoparticles by coacervation: implications in drug delivery. Trends Bio Artif Organs, 2005; 18(2):203-1.

Elshafey AH, Kamel AO, Awad GA. Ammonium methacrylate units polymer content and their effect on acyclovir colloidal nanoparticles properties and bioavailabilty in human volunteers. Colloids Surf Biointerfaces, 2010; 75(2):398-404.

Kalantzi LE, Karavas E, Koutris EX, Bikiaris DN. Recent advances in oral pulsatile drug delivery. Recent patents on drug delivery \& formulation, 2009 ; 3(1):49-63.

Matheson AJ and Jarvis B. Lansoprazole: an update of its place in the management of acid-related disorder. Drugs, 2001; 61(2):1801-33.

McConvillea JT, Rossa AC, Chambersa AR, Smithb G, Florencea AJ, Stevens HNE. The effect of wet granulation on the erosion behavior of an HPMC-lactose tablet, used as a rate-controlling component in a pulsatile drug delivery capsule formulation. European Journal of Pharmaceutics and Biopharmaceutics, 2004;57:541-549.

Paulo C, Jose M. Modeling and comparision of dissolution profiles, Eur J Pharm Sci. 2001; 13:123-133.

Santhi K, Dhanraj SA, Nagasamyvenkatesh D, Sangeetha S, Suresh B. Preparation and optimization of sodium alginate nanospheres of methotrexate. Indian J. Pharm. Sci., 2005; 67:691-696.

Schubert MA and Muler-Goyman CC. Solvent injection as a new aproach for manufacturing lipid nanoparticles-evaluation of the method and proces parameters. Eur J Pharm Biopharm, 2003; 5(1):12531.

Shimizu T, Nakano Y, Morimoto S, Tabata T, Hamaguchi N, Igari Y. Formulation study for Lansoprazole fast-disintegrating tablet. I. Effect of compression on dissolution behavior. Chem Pharm Bull, 2003;51(8):942-947.

Sonavane GS, Devarajan PV. Preparation of alginate nanoparticles using eudragit E100 as a new complexing agent development, in-vitro and in-vivo evaluation. Journal of biomedical nanotechnology, 2007; 3:160-169.

Sutch JCD, Ross AC, Ckenberger WK, Bowtell RW, MacRae RJ, Stevens HNE, Melia CD. Investigating the coating-dependent release mechanism of a pulsatile capsule using NMR microscopy. Journal of Controlled Release, 2003; 92: 341- 347.

Yung-Chih Kuo. Loading efficiency of stavudine on polybutylcyanoacrylate and methylmethacrylate sulfopropylmethacrylate copolymer nanoparticles. International Journalof Pharmaceutics, 2005; 290: 161-172.

How to cite this article:

Nagarajan E, Shanmugasundaram P, Ravichandiran. V, Vijayalakshmi A, Masilamani K. Development and Evaluation of Chitosan Based Polymeric Nanoparticles of an Antiulcer Drug Lansoprazole. J App Pharm Sci, 2015; 5 (04): 020-025. 